\title{
Assessing Pediatric Trauma Specimen Integrity
}

\author{
RUTH A. BUSH, TERESA MUELLER, BEVERLY SUMWALT, \\ SUSAN A. COX, MARY L. HILFIKER
}

\begin{abstract}
OBJECTIVE: This study investigated the quality of trauma specimens by comparing line draws to venipuncture.
\end{abstract}

DESIGN: The draw type (line or venipuncture); container type (Vacutainer or Microtainer); and suitability for processing (processed/hemolyzed/clotted) of routinely collected trauma specimens was analyzed.

SETTING: The clinical laboratory of a Level I Pediatric Trauma Center.

MAIN OUTCOME MEASURE: Hemolyzed trauma specimens were analyzed according to method of collection, collector, and type of container to identify issues resulting in unusable samples.

RESULTS: The data shows that for $13 \%$ of all draws, portions of the results were affected by hemolysis. Sixteen percent of line draws and $6 \%$ of venipunctures were hemolyzed $(p=0.04)$. There was no statistical association with who collected the sample $(p=0.07)$ or type of container $(p=1.00)$.

CONCLUSION: Based on this sample of data, the laboratory recommends that, whenever possible, venipunctures be performed for laboratory testing of blood specimens to improve trauma specimen integrity.

\section{ABBREVIATIONS:}

$\mathrm{ED}=$ Emergency Department; $\mathrm{EMS}=$ Emergency Medical Services; ID = identification; MLA = medical laboratory assistant; $\mathrm{RBC}=$ red blood cells; $\mathrm{RN}=$ registered nurse.

INDEX TERMS: hemolyzed specimens; specimen collection; evidence-based practice; trauma.

Clin Lab Sci 2010;23(4):219
Ruth A. Bush, PhD, Research Department, Rady Children's Hospital San Diego, San Diego, CA 92123

Teresa Mueller, CLS, Laboratory Department, Rady Children's Hospital San Diego, San Diego, CA 92123

Beverly Sumwalt, CLDir, Laboratory Department, Rady Children's Hospital San Diego, San Diego, CA 92123

Susan A. Cox, MS, Trauma Department, Rady Children's Hospital San Diego, San Diego, CA 92123

Mary L. Hilfiker, MD, PhD, Trauma Department, Rady Children's Hospital San Diego, San Diego, CA 92123

Address for Correspondence: Ruth A. Bush, Research Department (MC 5074), Rady Children's Hospital San Diego, 3020 Children's Way, San Diego, CA 92123, 858.966.4946,rbush@rchsd.org

Acknowledgements: Gary Melendrez, MLA, and Tony Mills, MLA, were integral in designing the study and collecting samples.

A high-functioning pediatric trauma team relies on the critical tool of rapid laboratory diagnostics. When blood specimens are unusable because of hemolysis, critical time and patient-care resources must be redirected to obtain repeat blood draws. In addition to time lost, within a pediatric population there is the additional risk of repeated blood collections resulting in iatrogenic anemia, ${ }^{1}$ as well as the anxiety of blood draws for both the child and the family.

The quality of patient specimens is an important determinant in laboratory testing. It has been estimated that $90-95 \%$ of diagnostic delays can be attributed to problems associated with error in the pre-analytic phase of laboratory medicine. ${ }^{2}$ In most clinical laboratories, 


\section{RESEARCH AND REPORTS}

hemolyzed specimens cannot be used for testing in blood bank, coagulation studies, and most chemistry procedures. ${ }^{3}$ Research is being undertaken to look at issues of specimen procurement and specimen handling to identify issues and to implement quality improvement methods. Factors of interest that result in hemolyzed specimens include: over-manipulation of the extremity at the draw site, intravenous catheter size, Vacutainer pressure, over-shaken or vigorously shaken tubes, utilization of an improperly sized tube or a tube without the proper additive, method of transport, and transport time to processing., ${ }^{3,4}$

Increased hemolysis rates, especially in hospital emergency departments (ED) have been attributed to the practice of obtaining blood specimens through intravenous catheters. To minimize patient discomfort and to save valuable clinical time for those patients requiring both an IV infusion and blood test, blood samples are often obtained through the IV catheter at the time of its insertion. ${ }^{5}$

This investigation explored the potential causes of specimen hemolysis by evaluating the procurement and handling of trauma patient specimens at a Level I Pediatric Trauma Center. Data derived from this investigation were used to compare catheter specimens to venipuncture specimens. Results of the study may help to identify whether procedural changes in specimen collection practices are needed to improve pediatric trauma specimen integrity.

\section{METHODS}

This research project was conducted as a prospective study from a nonconsecutive sample of pediatric trauma patients (aged 14 years and younger) in a 248-bed comprehensive acute and tertiary care medical center, which includes a Level I Pediatric Trauma Center. The institutional review board at Rady Children's Hospital approved this study protocol. [Data collection was exempt from requiring written informed consent as stipulated in the Department of Health and Human Services regulation 45 CFR 46, Nos. 3 and 5, because data were collected from a database that already existed and no participant was contacted for the study.] Candidates for study inclusion were those patients who required a blood draw for laboratory diagnostics. There were no changes in the standard of care for blood specimens obtained from patients included in the study.

The blood collector group consisted of 70 experienced ED nurses (RN) and 17 medical laboratory assistant (MLA) phlebotomists. The determination to collect blood via venipuncture versus IV was based upon whether the patient presented with an existing intravenous catheter (placed prior to arrival by Emergency Medical Services personnel) and the requirement to initiate IV line insertion. The choice of IV catheter size and venipuncture site were at the RN/MLA's discretion. For those individuals with an IV, the lines were cleared before obtaining blood according to standard operating procedure and 3 to $10 \mathrm{~mL}$ syringes were used to aspirate the blood. Blood samples obtained by venipuncture were drawn through a 21 -, 22-, 23-, or 25-gauge needle. With both types of draws, blood was subsequently transferred from the syringe to Vacutainers using a blood transfer device or directly to Microtainers. Selection of blood container type was based upon the volume of blood obtained and the patient's condition.

After obtaining blood specimens, the MLA assigned to the trauma documented patient identifiers; specimen accession numbers; method of collection; whether the collector was an RN or MLA; and type of container on a standardized trauma specimen Study Log. The medical laboratory technologists performing the tests documented on the laboratory report whether specimens were clotted, hemolyzed, or of insufficient volume.

Medical laboratory technologists assessed plasma and serum for hemolysis by comparison to a standardized visual hemolysis chart. According to laboratory protocol, a hemolysis level corresponding to an approximate hemoglobin concentration below 100 $\mathrm{mg} / \mathrm{dL}$ is judged to be acceptable for testing. Hemolyzed specimens corresponding to approximate hemoglobin concentrations above $100 \mathrm{mg} / \mathrm{dL}$ are not analyzed. Additionally, coagulation was defined as visible evidence of fibrin formation.

All statistical analyses were performed with SPSS software (Release 17.0; SPSS Inc. Chicago, IL). Chi- 


\section{RESEARCH AND REPORTS}

squared and cross tabs analysis were completed. Tests were considered significant if $P$ values were less than 0.05 .

The total number of hemolyzed specimens received by the laboratory were evaluated. Data collected over the initial eight-month period were evaluated by totaling the number of specimens received (affected and nonaffected) and assigning percentages of specimens hemolyzed based on type of draw, container, and phlebotomist. The trends in the data indicated that it would be useful to conduct a second ten month phase of the study to provide increased statistical power. The additional data would also help to eliminate possible seasonality associated with type of trauma and variations in staffing. The eighteen months of pooled data were analyzed to determine if those three variables were associated with the occurrence of hemolyzed specimens.

\section{RESULTS}

Blood draw information for 221 individuals were analyzed. The study was conducted in two phases; October 2006 through May 2007 (110 individuals) and August 2008 through May 2009 (111 individuals). Of the 221 blood draws, 30\% $(\mathrm{n}=66)$ were by new venipuncture and $70 \%(\mathrm{n}=155)$ were through existing intravenous sites. Approximately 13\% ( $\mathrm{n}=29)$ of the samples had some level of hemolysis and approximately $2 \%(\mathrm{n}=5)$ had clotting. Differences emerged when results were further analyzed with respect to method of blood draw (Table 1). Sixteen percent of the line draws had some degree of hemolyzation $(\mathrm{n}=25)$ versus $6 \%$ ( $\mathrm{n}$ = 4) for venipuncture samples. A Pearson Chi-Squared demonstrated an association between line draws and hemolyzed samples $(p=0.04)$ in this sample. Additional analysis examining whether the specimen was obtained by a nurse or a phlebotomist was conducted. Although $83 \%(\mathrm{n}=24)$ of the hemolyzed samples were obtained by a nurse, a Pearsons Chi-Squared did not demonstrate a statistical association with collector and hemolyzed sample $(p=0.07)$.

\section{DISCUSSION}

The American Society of Clinical Pathology has identified the benchmark hemolysis rate of $2 \%$ or less for collection of blood samples as best practice (4). While national programs to track laboratory quality have reported aggregate specimen rejection rates of $0.83 \%$ in hospital-based laboratories, rates for hemolyzed specimens, especially in Emergency Departments are much higher and are the primary reasons for specimen rejection. ${ }^{2}$ Other studies have reported rates of $13 \%$ to $32 \%,{ }^{5,6}$ which are similar to this study and suggest inherent issues in a rapid blood draw. It has been noted that EMS patients who arrive with intravenous lines are more likely to have a line draw than additional venipuncture. ${ }^{4}$ As many ED and trauma patients have pre-hospital medical contact, existing catheters are used with a high number of samples obtained by line draw.

Table 1: Unusable Samples by Location, Container, and Collector $(\mathrm{N}=221)$

\begin{tabular}{|c|c|c|c|}
\hline & Method & Total $(\%)^{*}$ & Hemolyzed (\%) \\
\hline & Venipuncture $^{* *}$ & $66(30 \%)$ & $4(2 \%)$ \\
\hline & Line draw & $155(70 \%)$ & $25(11 \%)$ \\
\hline & Microtainers $\dagger$ & $11(5 \%)$ & $1(0 \%)$ \\
\hline & Vacutainers & $199(95 \%)$ & $23(10 \%)$ \\
\hline & $\mathrm{RN} \ddagger$ & $151(68 \%)$ & $24(11 \%)$ \\
\hline & MLA & $70(32 \%)$ & $5(2 \%)$ \\
\hline . & \multicolumn{3}{|c|}{ Percent of total sample } \\
\hline ** & \multicolumn{3}{|c|}{ Pearson Chi-Squared $=4.12 ; p=0.04$} \\
\hline$\dagger$ & \multicolumn{3}{|c|}{$\begin{array}{l}\text { Microtainer and Vacutainer were excluded from the container } \\
\text { analysis; Fisher Exact Test }-p=1.00\end{array}$} \\
\hline$\ddagger$ & \multicolumn{3}{|c|}{ Pearson Chi-Squared $=3.21 ; p=0.07$} \\
\hline
\end{tabular}

The issue of hemolysis of blood samples and the need for re-collection of laboratory specimens, particularly in Emergency Departments, has been examined in the adult population. ${ }^{3-5}$ Various research approaches retrospective, case/control, and prospective randomization have found that blood samples obtained by venipuncture rather than intravenous catheter were significantly less likely to result in hemolysis. ${ }^{2,4,6}$ Lowe et al. have gone so far as to suggest that in the ED collection of specimens by venipuncture should be considered standard of care. ${ }^{4}$ To our knowledge, this is 


\section{RESEARCH AND REPORTS}

the first study in a pediatric population to suggest similar results.

Although some adult specimen integrity studies restricted obtaining the blood draw to nurses, ${ }^{4,5}$ other methodology has suggested that certified laboratory phlebotomists are more likely than ED RNs to collect usable specimens. ${ }^{3}$ Data from this pediatric study was able to compare the outcome of phlebotomists to nurses. There was no statistical association with the type of the person who drew the specimen and the usability of the specimen. This result is important as the hospital's protocol restricts line draws to RNs and there could easily have been a confounded association between RNs and unusable samples since line samples were more likely to be hemolyzed.

The strengths of this study include inclusion of a pediatric population, who are generally excluded from such studies. Additionally, specimen integrity data were collected as part of the standard operating procedure and not prejudiced by knowledge of being part of a study or other influences that would have resulted in altered behaviors. There were limitations to the study including the fact that it was a nonrandomized convenient sample. Intravenous catheter diameter, which has emerged as an important variable in other studies (5), was not analyzed in this study. In the future, it may be helpful to look at the interaction between syringe and needle size, as well as technique employed when filling the syringe to examine the outcomes on hemolyzed collections. While certain components of the phlebotomy process such as tourniquet time and time from specimen collection to analysis were not collected, the fact that all of the samples were collected by the same staff and all were run "stat" should have reduced variability and meaningful analysis among these metrics. Mode of transport was consistent.

\section{CONCLUSION}

The outcomes of this study suggest that a policy development specifying practice guidelines recommending that, whenever possible, venipunctures be performed for laboratory testing of blood specimens from pediatric trauma patients to improve trauma specimen integrity. Future research in this area could include intravenous catheter size and hemolysis rates for a larger sample size similar to that obtained in the study and randomization to one or other standardized protocols for a more rigorous analysis.

\section{REFERENCES}

1. Park YA, Marques MB. Teaching Medical Students Basic Principles of Laboratory Medicine Clin Lab Med - 2007 Jun; 27 (2): 411-24.

2. Stark A , Jones BA, Chapman D, Well K, et al. Clinical Laboratory Specimen Rejection-Association With the Site of Patient Care and Patients' Characteristics: Findings From a Single Health Care Organization. Arch Pathol Lab Med 2007 Apr; 131 (4): 588-92.

3. Pretlow L, Gandy T, Kenimer Leibach E, Russell B, Kraj B.A Quality Improvement Cycle: Hemolyzed Specimens in the Emergency Department. Clin Lab Sci 2008; 21(4): 219-24.

4. Lowe G, Stike R, Pollack M, Bosley J, O'Brien P, et al. Nursing blood specimen collection techniques and hemolysis rates in an emergency department: analysis of venipuncture versus intravenous catheter collection techniques. J Emerg Nurs. 2008 Feb;34(1):26-32. Epub 2007 Sep 25.

5. Kennedy C, Angermuller S, King R, Noviello S, Walker J, et al. A comparison of hemolysis rates using intravenous catheters versus venipuncture tubes for obtaining blood samples. J Emerg Nurs. 1996 Dec;22(6):566-9.

6. Grant MS. The effect of blood drawing techniques and equipment on the hemolysis of ED laboratory blood samples. J Emerg Nurs. 2003;29(2):116-21. 